This document is the accepted manuscript version of the following article:

Larsen, T. A., Gebauer, H., Gründ1, H., Künzle, R., Lüthi, C., Messmer, U., ... Ranner, B. (2015). Blue Diversion: a new approach to sanitation in informal settlements. Journal of water Sanitation and Hygiene for Development, 5(1), 64-71.

https://doi .org/10.2166/washdev.2014.115

BLUE DIVERSION:

\title{
A NEW APPROACH TO SANITATION IN INFORMAL SETTLEMENTS
}

Tove A. Larsen ${ }^{\mathrm{a},{ }^{*}}$, Heiko Gebauer ${ }^{\mathrm{a}}$, Harald Gründl ${ }^{\mathrm{b}}$, Rahel Künzle ${ }^{\mathrm{a}}$, Christoph Lüthia ${ }^{\mathrm{a}}$, Ulrike Messmer $^{\mathrm{a}}$, Eberhard Morgenroth ${ }^{\mathrm{a}, \mathrm{c}}$, Charles B. Niwagaba $^{\mathrm{d}}$, Bernhard Ranner ${ }^{\mathrm{b}}$

${ }^{a}$ Eawag, Swiss Federal Institute of Aquatic Science and Technology, 8600 Dübendorf, Switzerland; ${ }^{\mathrm{b}}$ EOOS, Zelinkagasse 2/6, 1010 Vienna, Austria

${ }^{\mathrm{c}}$ Institute of Environmental Engineering (IfU), ETH Zurich, 8093 Zurich.

${ }^{\mathrm{d}}$ Department of Civil and Environmental Engineering, Makerere University, P.O. BOX 7062, Kampala, Uganda

${ }^{*}$ Corresponding author: Tel. +41 58765 5039; email: tove.larsen@eawag.ch

\section{ABSTRACT}

The sanitation concept 'Blue Diversion' (www.bluediversiontoilet.com) was developed as a possible answer to the sanitation crisis in urban slums. The concept is based on two main elements: (1) diversion of urine, feces, and water at the source as the basis for efficient resource recovery, and (2) linking different scales (household level and semi-centralized resource recovery) to allow rapid implementation of family toilets. Our objective was to develop an attractive "grid-free" dry diversion toilet (separately diverting undiluted urine and feces) which provides water (through recycling of water on-site) for flushing, personal hygiene (anal cleansing and menstrual hygiene), and hand washing. This service, including the entire sanitation value chain, should eventually be made available as a profitable business with total user fees of $5 \phi / \mathrm{p} / \mathrm{d}$. The results presented in this paper are (1) a toilet design model, (2) the development of a new type of membrane bioreactor for 
treating flush \& wash water, (3) main results of a GIS-based stochastic service model to link the family scale toilet to a community-scale Resource Recovery Plant, and (4) a business model that yield maximum profit for the local community. We conclude that the approach is feasible, but challenging.

Keywords: Toilet design, innovation, resource recovery, source separation, urban, wastewater treatment

\section{ABBREVIATIONS}

BMGF: Bill \& Melinda Gates Foundation

RRP: Resource Recovery Plant

RTTC: Reinvent The Toilet Challenge (a call from the BMGF)

UDDT: Urine Diverting Dry Toilet

UF: Ultra Filtration

\section{INTRODUCTION}

In 2010 , around $10 \%$ of the deaths occurring in children below the age of five could be attributed to diarrhea (Liu et al., 2012). This corresponds to more than 800’000 deaths a year, with the highest numbers in Africa and Southeast Asia. Hygienic sanitation and hand washing can reduce the incidence of diarrhea significantly (see e.g. Curtis \& Cairncross 2003; Fewtrell et al. 2005). It is well-known that the MDG goals for improved sanitation will not be reached, but as pointed out by Baum et al. (2013) the situation may in fact be even worse than normally anticipated. The reason is 
that "improved sanitation" is defined only with respect to personal hygiene, while all aspects of urban hygiene, which would include safe disposal or treatment of excreta, are neglected.

This may be one of the reasons that the Bill \& Melinda Gates Foundation (BMGF) launched the Reinvent The Toilet Challenge (RTTC) in 2011 (Anonymous 2011). Although most of the BMGF sanitation grants are directed towards projects following the state-of-the-art for sanitation projects in low-income countries, the foundation also wanted to challenge other parts of the scientific community to invent unconventional 'high-tech' solutions.

The requirements of the call were ambitious: to develop a private or public toilet for dense urban areas (especially informal settlements), providing high personal comfort at a price of $5 \varnothing$ per person and day. Valuable products should be extracted from the toilet waste, and no connection to running water, grid electricity, or sewers could be assumed. In essence, there was a requirement for 'zeroemission', which would take into account urban hygiene as discussed above as well as environmental aspects.

A total of eight projects were initiated (including one from our institution), ranging from electrochemical treatment of combined toilet waste to plasma gasification of source separated feces (Anonymous 2011). As opposed to other grantees, we chose to focus on toilet design and logistics and not on processing excreta. We based this choice on compelling evidence that in many developing countries toilets are of poor quality, not only from the perspective of user satisfaction (see e.g. Tumwebaze et al. 2012), but also with respect to the entire sanitation value chain (Evans 2013). Our approach, 'Blue Diversion', discussed in this paper, was based on two main concepts: (1) separation of urine, feces, and water for better resource recovery in the sanitation value chain 
and (2) adaptation of two different scales: the household scale for the toilet and a semi-centralized scale for treatment and resource recovery.

Source separation is a relatively new field in urban water management (Larsen et al. 2013), but especially in developing countries, this idea is gaining ground (see e.g. Katukiza et al. 2012 for an overview). The main advantage of source separation is an increased resource efficiency as discussed by Larsen et al. (2009). It is e.g. more energy-efficient to extract valuable resources from a concentrated 'pure' stream (like urine or feces) than from a diluted mixture (e.g. of excreta and flush water). Urine diverting dry toilets (UDDTs), however, are often not well accepted by users, especially in an urban environment (Cordova and Knuth 2005). Since they cannot be flushed, they tend to end up dirty and unattractive, and the lack of water for hand washing and anal cleansing (for washers) reduces the hygienic improvements achieved by these toilets. For this reason we chose to develop an attractive source-separating dry toilet with an integrated water cycle.

Recovery of resources from urine and feces are easier from a toilet with many users, but as shown by Tumwebaze et al. (2012), user satisfaction is negatively correlated with the number of users per toilet. For this reason, we chose different scales for toilet and resource recovery, and linked those by a simple transport system as shown in Figure 1. We considered that the main concerns about transport are related to hygiene and aesthetics for the people emptying the toilets, whereas costs are the main issue from a business point of view. We thus focused on these two aspects.

In this paper, we present key features of our approach to reinvent a toilet, which can be integrated into a sanitation value chain in informal settlements. Our main focus is on toilet design and toilet technology, which are the most innovative parts of the project. 


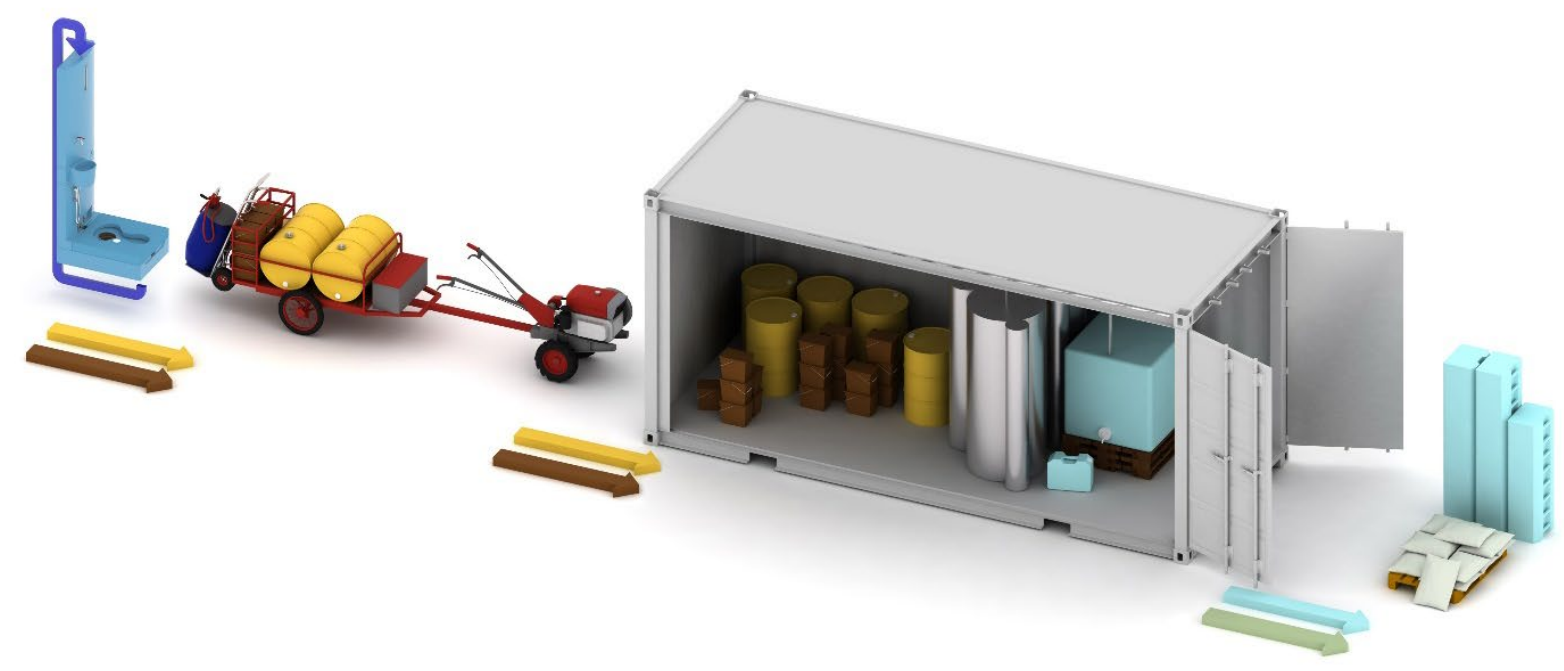

Figure 1 Conceptual set-up of the Blue Diversion system with the following features: (1) an attractive source-separating toilet (first model 2012); (2) wash \& flush water is recovered and recycled on-site; (3) a concept for transport is set up; (4) semi-centralized resource recovery from source-separated urine and feces; (5) a market-based business model. An important source of income may arise from the sale of fertilizer chemicals extracted from urine.

\section{METHODS}

The project was developed in an interdisciplinary team (engineering, social science, business administration, sanitation in developing countries, and design). We also involved different stakeholders in workshops and focus groups, based on good practice for implementation of sustainable sanitation in developing countries (Lüthi et al. 2011).

The design process was initially supported by three workshops: (1) an internal Eawag workshop, additionally attended by two international experts; (2) a workshop at the Makarere University (Department of Civil and Environmental Engineering), and (3) a local workshop in an informal 
settlement in Kampala, Uganda. Furthermore, a research log was set up to document the state-ofthe-art in the area of UDDTs (EOOS \& WEDC 2014). A first working model was pilot tested in Uganda (Tobias et al. subm.) and the subsequent working model (after modification) in Kenya (unpublished data).

The development of the water recovery unit was initiated by a number of internal Eawag workshops, where technical alternatives were developed by different groups of engineers. We aimed for a high diversity in the groups with respect to age, competences, and experience. In a final workshop, the technical alternatives were evaluated and the most promising ones were tested. For more detailed methodology of the testing, see Künzle et al. (subm.).

In order to set up the transport concept, a numerical model was developed in order to evaluate and optimize transport in a variety of spatial set-ups (Schmitt 2012). We used GIS data for the housing structures and road networks of four informal settlements in Africa and India. Regression techniques were used to analyze the spatial data (especially the influence of population density on travel distances). Moreover, bivariate regressions were applied in order to identify the interdependencies of the system parameters, e.g. of service time on costs and capacity.

Different business model solutions were developed based on a one-day, participatory workshop with participants from different disciplines (from Eawag and ETH Zürich) and one PhD student from Kampala, Uganda. The workshop was inspired by the BOP (base of the pyramid) protocol 2.0 (Simanis and Hart 2008), but only covered the first, qualitative part of the process. 


\section{RESULTS}

\section{Attractive source-separating toilet}

A major goal of the Blue Diversion project was to design an attractive source-separating toilet that will appeal to users. Based on the preferences of the participants in the local Kampala workshop, we chose to design a squatting toilet, but the same principles can be used for a sitting toilet. In order to counteract the lack of hygiene associated with dry sanitation (Katukiza et al. 2012), the toilet provides running water for hand washing and personal hygiene, and a comfortable "flush" button for cleansing the front part of the pan. These familiar functions are translated into a "next generation" product that works off-grid by applying a radical low-cost innovation approach. An important feature of this low-cost approach is the fact that most parts of the toilet can be produced locally in simple processes, e.g. rotational molding.

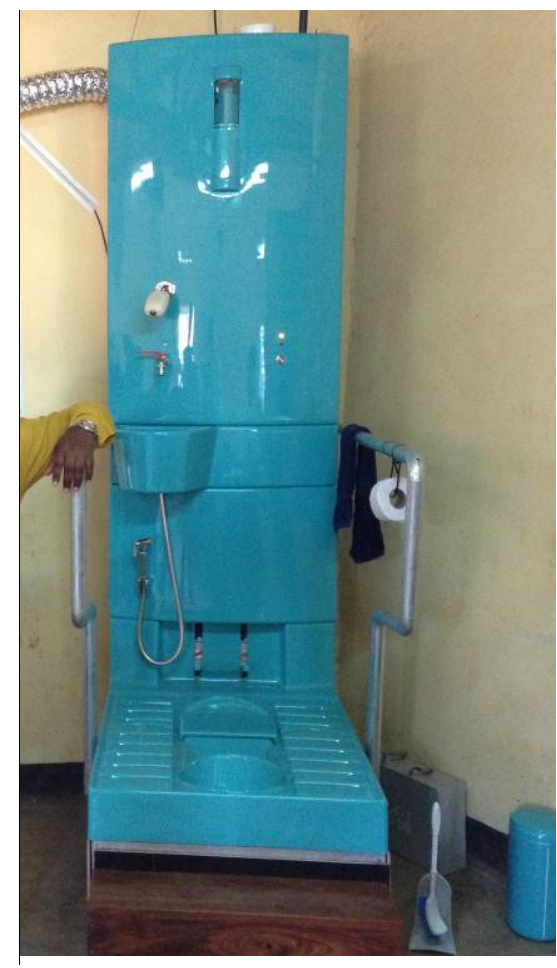

(a)

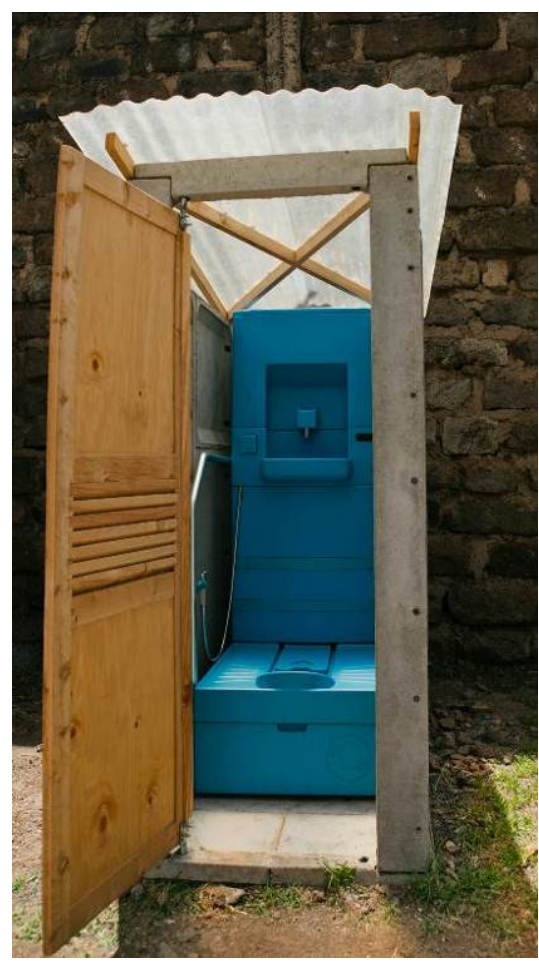

(b)

Figure 2 Working models of Blue Diversion toilet: (a) the first working model tested in Uganda; (b) the second working model tested in Kenya. 
The toilet is laid out for the use by approximately 10 people as a compromise between acceptance, size and costs. Tumwebaze and Mosler (2014) investigated the risk of a social dilemma when it comes to the cleaning of shared toilets in urban slums. According to the Water Supply and Sanitation Collaborative Council's proposed WASH post-2015 targets, a toilet can be considered as acceptable or improved if it is shared by maximum five families. However, as the risk of a social dilemma in the collective cleaning behavior of a shared toilet declines with the number of families sharing the toilet, we defined the Blue Diversion Toilet for the scale of two moderately sized families.

As a (re)movable piece of furniture, it can be retrofitted into existing toilet superstructures or fitted in any bathroom, thus allowing a "rent-a-toilet" system to be set up. This retrofitting concept as well as the toilet functions and aesthetics were tested in an initial reality-check workshop in an informal settlement in Kampala, where the concept was well received.

The toilet features an innovative dry source separating pan where the front part can be cleaned with water from the on-site water recovery unit. The toilet's core is the back wall containing the compact water recovery technology. While feces and urine are collected under the separating pan (like in a normal UDDT), the polluted water from hand-washing, cleaning of the front-end of the pan, anal cleansing and menstrual hygiene is fully recycled on-site. Important design features of the water recovery wall are a hand wash basin with running water, and a shower head providing a comfortable cleansing device for personal hygiene (Figure 2). The feces cover and a specially developed urine-water separator (hidden below the pan) are both activated by the flush and the hand shower in order to prevent mixing of excreta and water (see www.bluediversiontoilet.com for technical details). 


\section{On-site water recovery}

The core principle of the on-site water recovery technology is a gravity-driven ultrafiltration (UF) unit originally developed for obtaining drinking water from river water (Peter-Varbanets et al. 2009). The advantage of this technology is the ability to produce hygienically safe water (by ultrafiltration) at low energy consumption (filtration is entirely gravity-driven) and without maintenance (in the low-loaded filter, grazing by higher organisms keeps the filter open; Derlon et al. 2012). Due to the more heavily polluted wastewater to be recovered within the toilet (as compared to river water) and recirculation of the treated water, modifications to the original concept were necessary. Künzle et al. (subm.) showed in experiments with simulated flush \& wash water polluted with feces, urine, soap, and blood that a stable flux could be obtained with the simplest possible reactor, contained within the water recovery wall (Figure 2). The key technical design parameters for sizing the reactor are the water permeability and the trans-membrane pressure, as these determine the water flux. Initial results confirm that about 75 liters of water can be made available per day (corresponding to 1.5 liters of water per toilet visit, assuming 10 users and 5 toilet visits per user and day).

As the filtered water contains around 30-40 $\mathrm{mg} \mathrm{COD} / \mathrm{L}$ and is slightly colored, we recommend polishing of the effluent to minimize the risk of re-growth and ensure color removal. Preliminary results with chlorine-producing electrolysis were successful (Künzle et al. subm.), and an energyoptimized version of electrolysis functioned well in the second field-test in Kenya (unpublished data).

We found that safe water recovery through gravity-driven UF is feasible and maintenance-free operation is stable. Polishing by electrolysis makes the water more acceptable and increases the 
safety level. Energy consumption could be optimized to around $30 \mathrm{Wh} / \mathrm{p} /$ day, which can be provided by a small solar panel and battery.

\section{Transport logistics concept}

The conceptual transport logistics comprise one collector per Resource Recovery Plant (RRP), collecting feces and urine twice a week from the toilets. In order to ensure hygienic and aesthetic working conditions, feces are removed in a self-sealing container and urine by pumping. These features, however, still only exist as design studies (not presented).

We explored whether a collection service system would be feasible at a reasonable price, despite labor-intensive collection in complex contexts. Analysis of the spatial data showed that travel distances are significantly related to user density. However, even at moderate densities from 100 350 users per hectare, this has only a minor influence on the system performance. Instead work productivity was identified as the key driver for efficiency (Schmitt 2012). At lower user densities, travel distance plays an increasing role and at very high population densities, space will clearly limit the applicability of the concept, e.g. in Bangladesh, where Angeles et al. (2009) found population densities up to 2'550 inhabitants per hectare. This implies that the logistics system is suitable for a range of typical spatial set-ups, but only provided that work productivity is high and space can be found for toilets and treatment facilities. We conclude that one of the most important system integration parameters is the optimization of the feces collection process, urine pumping and discharge to the RRP, all features strongly influenced by design.

For the calculation of a business case, we chose Kampala in Uganda, with a user density of 150 inhabitants per hectare and a service time per facility of 20 minutes, resulting in an RRP for 860 users and logistic costs of $1 \not /$ user/day (Schmitt 2012). 


\section{Semi-centralized resource recovery from source-separated urine and feces}

Many technologies for treatment of source-separated feces and urine are known, but most of them are still in their infancy (for comprehensive presentations of many of these possibilities, see Larsen et al. 2013). The optimal choice of technology in a Resource Recovery Plant depends on the boundary conditions of a specific site and further technology development (for a discussion, see McConville et al., subm.).

\section{The Blue Diversion business model - a market-based sanitation approach}

The Blue Diversion business model follows the marked-based sanitation approach (Osterwalder and Pigneur 2010; London and Hart 2010). Sanitation entrepreneurs would generate sufficient revenues to cover the costs and obtain an attractive profit. This business model addresses two different customer groups: two families (average five persons per family) renting a single toilet and wholesalers buying end-products. The value proposition for toilet users is about offering attractive and affordable rental toilets as well as safe and reliable emptying; the value proposition for wholesalers is based on the secure delivery of nutrients or fertilizers at a stable price (independent of market fluctuations).

In the Blue Diversion business model, the relationship with the toilet users is managed via mobile payments and twice-a-week visits by the collector to remove feces and urine. Efficient treatment and resource recovery create another stream of revenue from the sales of end-products. These two revenue streams make the business model robust and assure a positive cash flow while still allowing for the provision of attractive toilets at an affordable service fee. The corresponding revenue streams are the service fee of $5 \phi$ per user and day, which covers toilet rent, emptying, and maintenance costs, as well as most of the RRP costs. Sales of end-products such as recovered water, nutrients, and energy are estimated to reach about $2 \varnothing$ per user and day. 
Key competencies for executing the business model include logistics, operational, and management skills. Logistics skills ensure the safe and hygienic removal of excreta, operational skills are necessary to convert urine and feces in the RRPs into valuable end-products, and management skills promote and market the "rent-a-toilet" service. Entrepreneurial skills to develop and scale-up the business model are another key element.

Necessary business partners are local toilet manufacturers, who produce the user interface, ultrafiltration membrane manufacturers, treatment technology equipment providers, who provide the RRP equipment, and finally, local contractors, who assemble and retrofit the toilet into the existing superstructure and provide the service of repairing the toilets and RRP machinery. Finally, to make the business model successful, the local community has to support the development of the sanitation business.

\section{Pilot testing of the Blue Diversion toilet}

A first model of the Blue Diversion toilet was tested in two slums (Kifumbira and Kisalosalo) in Kampala, Uganda (Tobias et al. subm.). Although the toilet was generally well received, a number of critical points were identified by the users. Improving on these issues gave rise to the second working model, which was field-tested in Mukuru, an informal settlement in Nairobi, Kenya, in cooperation with Sanergy (http://saner.gy/). Although the data have not yet been analysed in detail, it was clear that this second generation toilet was better received than the first one, showing the importance of rapid field testing and short development periods. From a technical point of view, however, there is still much work to be done in order to obtain the robustness required for largescale implementation. 


\section{DISCUSSION}

The sanitation solution presented in this paper is innovative, but it builds on many existing sanitation solutions. Both the UDDT and the pour flush toilets, where small amounts of water for flushing is used, are common toilet types in many low-income countries (Katukiza et al. 2010). Transporting feces and urine over short distances to a semi-centralized plant where resources are extracted is also done in other projects, e.g. by Sanergy (http://saner.gy/) and the idea of mobile toilet units is applied for instance in the MobiSan approach (Naranjo et al. 2010).

However, the Blue Diversion Toilet design combines those features and can thus be considered a further development of the UDDT: (1) the toilet can be flushed without water entering the urine or feces container; (2) the feces container is self-sealing, posing no risk for the service personnel (not yet developed); (3) water for hand washing, anal cleansing, and flushing is recovered and reused for the same purpose on-site; (4) washers do not have to displace themselves for anal cleansing; (5) a hand washing facility is included in the toilet design.

Although the toilet has already been field-tested twice in order to test for general acceptance especially for the acceptance of reuse of treated water for personal hygiene - it is still far from an industrialized product. Technical robustness, willingness to pay, and acceptance of the features are only a few of the many barriers to a successful introduction of this new toilet design. These features can be tested and improved in small pilot projects. For the toilet to be applied at scale, however, the business model must also be refined and successfully implemented. The low fee of $5 \phi / \mathrm{p} / \mathrm{day}$ is based on large-scale production of all parts of the toilet and marketing of the fertilizer chemicals produced. Important next steps are thus: 
1. Technical progress to improve robustness and drive down potential costs of mass production. Some parts of the toilet have not been developed yet; other parts like the solar panel depend on other industrial developments in order to bring the costs down.

2. Further field testing in diverse environments (for acceptance and robustness).

3. Further development of the business model and cooperation with organizations already working in the field along the same lines.

Despite the potential advantages of the Blue Diversion Toilet, there is still a long way to go before the concept can be implemented at scale. Considerable investments must be made over years in order to make this apparently high-end toilet available for the poor at a price that they are both able and willing to pay.

\section{CONCLUSION}

We conclude that

- The systems approach presented in this paper is based on existing concepts, but improves on critical issues like water availability and acceptance.

- It is possible to optimize the single components of the system, provided that the right competences are available.

- Setting up a robust technical system is necessary, but not enough. Only if the technology is integrated into an equally robust business model and grounded in reality will the system succeed. This is in our opinion feasible, but challenging. 


\section{ACKNOWLEDGEMENTS}

This project was financed by the Bill \& Melinda Gates Foundation. We thank a large number of colleagues and potential users at and outside of Eawag and the University of Makarere for participating in our workshops, and the colleagues at Sanergy for invaluable support during the second field-test in Kenya.

The business model was funded by the Swiss Agency for Development and Cooperation.

\section{REFERENCES}

Robson, A. J., Jones, T. A. \& Reed, D. W. 1998 A study of national trend and variation in UK floods. Int. J. Climatol. 18, 165-182.

Angeles G., Lance P., Barden-O’Fallon J., Islam N., Mahbub A. Q. M. \& Nazem N. I. 2009 The 2005 census and mapping of slums in Bangladesh: design, select results and application. Int. J. Health Geogr. 8(1), 32-51.

Anonymous 2011 tce process news: Gates offers grants to redesign the toilet. Chemical Engineer 843, 18-19.

Baum, R., Luh, J. \& Bartram, J. 2013 Sanitation: A global estimate of sewerage connections without treatment and the resulting impact on MDG progress. Environ. Sci. Technol. 47(4), 19942000. 
Cordova A. \& Knutz B. A. 2005 Barriers and strategies for dry sanitation in large-scale and urban settings. Urban Water J. 2(4), 245-262

Curtis, V. \& Cairncross, S. 2003 Effect of washing hands with soap on diarrhoea risk in the community: A systematic review. Lancet Infect. Dis. 3(5) 275-281

Derlon N., Peter-Varbanets M., Scheidegger A., Pronk W. \& Morgenroth E. 2012 Predation influences the structure of biofilm developed on ultrafiltration membranes. Water Res. 46(10), 3323-3333.

EOOS \& WEDC 2014 A collection of contemporary toilet designs. Loughborough, UK. http://wedc.lboro.ac.uk/resources/books/Contemporary_Toilet_Designs.pdf

Evans B. 2013 Cities of the global South - is decentralized sanitation a solution? In: Source Separation and Decentralization for Wastewater Management, Larsen T. A., Udert K.M. \& Lienert J. (eds), IWA Publishing, London - New York, pp. 117-132.

Fewtrell, L. , Kaufmann, R.B., Kay, D., Enanoria, W., Haller, L. \& Colford Jr., J.M. 2005 Water, sanitation, and hygiene interventions to reduce diarrhoea in less developed countries: A systematic review and meta-analysis (Review). Lancet Infect. Dis. 5(1), 42-52.

Günther, I., Horst, A., Lüthi, C., Mosler, H.J., Niwagaba C.B. \& Tumwebaze, I.K. 2012 When is shared sanitation improved sanitation? Research for Policy2. Eawag. Switzerland 
Katukiza A.Y., Ronteltap M., Niwagaba C. B., Foppen J. W. A., Kansiime F. \& Lens P. N. L. 2012 Sustainable sanitation technology options for urban slums. Biotechnol. Adv. 30, 964-978.

Katukiza A.Y., Ronteltap M., Oleja A., Niwagaba C. B., Kansiime F., Lens P. N. L. 2010. Selection of sustainable sanitation technologies for urban slums - A case of Bwaise III in Kampala, Uganda. Sci. Total Environ. 409, 52-62.

Künzle R., Pronk W., Morgenroth E. \& Larsen T. A. subm. An energy-efficient membrane bioreactor for on-site treatment and recovery of wastewater. Subm. to J. Water, Sanitation Hyg. Dev.

Larsen T. A., Alder A. C., Eggen R. I. L, Maurer M. \& Lienert J. 2009 Source separation: Will we see a paradigm shift in wastewater handling? Environ. Sci. Technol. 43(16), 6121-6125.

Larsen T. A., Udert K. M. \& Lienert, J. (eds.) 2013 Source Separation and Decentralization for Wastewater Management. IWA Publishing, London, UK.

Liu, L. \& 11 further authors 2012 Global, regional, and national causes of child mortality: an updated systematic analysis for 2010 with time trends since 2000. Lancet 379(9832), 2151-2161.

London T. \& Hart S. 2010 Next Generation Business Strategies for the Base of the Pyramid: New Approaches for Building Mutual Value. Financial Times Press, Upper Saddle River, NJ.

Lüthi C., Panesar A., Schütze T., McConville J., Parkinson J. \& Saywell D. 2011 Sustainable Sanitation in Cities: A Framework for Action. Papiroz Publishing, The Netherlands. 
McConville, J.R., Kuenzle, R., Messmer, U., Udert, K.M., Larsen, T.A. subm. Reinventing the toilet: Structuring the design space for innovative treatment technologies. Submitted to Environ. Sci. Techn.

Naranjo A., Castellano D., Kraaijvanger H., Meulman B., Mels A. \& Zeeman G. 2010 The MobiSan approach: informal settlements of Cape Town, South Africa. Water Sci. Technol. 61(12), 3078-3090.

Osterwalder A. \& Pigneur Y. 2010 Business Model Generation: A Handbook for Visionaries, Game Changers, and Challengers. John Wiley \& Sons, New Jersey.

Peter-Varbanets M. Zurbrügg C., Swartz C. \& Pronk W. 2009. Decentralized systems for potable water and the potential of membrane technology. Water Res. 43(2), 245-265.

Schmitt R. 2012 Reinvent the Toilet Challenge. Validation of the transport logistic concept. Project Report, Eawag (available from corresponding author on request).

Simanis E. \& Hart S. 2008 The Base of the Pyramid Protocol: Toward Next Generation BoP Strategy. 2nd edn, Cornell University.

Tobias, R., O’Keefe, M., Künzle, R., Gebauer, H., Gründl, H., Pronk, W., Morgenroth, E. \& Larsen, T.A. subm. Applying social-science methods for co-designing a new toilet for slum areas. Submitted to Environ. Sci. Techn. 
Tumwebaze, K. I. \& Mosler H.-J. In press. Why clean the toilet if others don't? Using a social dilemma approach to understand users of shared toilets' collective cleaning behaviour in urban slums: a review. J. Water, Sanitation Hyg. Dev. 